\title{
Implicaciones psiquiátricas y neurológicas en la literatura shakespeariana. Breve análisis
}

\author{
Julio César López-Valdés, ${ }^{1}$ Alejandro Miranda-Hernández² y Jorge Alberto Medina-Medina ${ }^{3}$ \\ ${ }^{1}$ Universidad Autónoma de Tamaulipas, Facultad de Medicina de Tampico, Tamaulipas, México; ${ }^{2 H o s p i t a l ~ P r i v a d o, ~ C i u d a d ~ d e ~ M e ́ x i c o, ~ M e ́ x i c o ; ~}$ \\ ${ }^{3}$ Instituto de Seguridad y Servicios Sociales de los Trabajadores del Estado, Hospital Regional 1 de Octubre, Ciudad de México, México
}

\author{
Diseases desperate grown, \\ By desperate alliances are relieved, \\ Or not at all. \\ Hamlet, Acto Iv, Escena 3
}

\section{Resumen}

William Shakespeare (1564-1616) es sin duda uno de los artistas literarios más prolificos de todos los tiempos. Además de ser un dramaturgo eminente, en sus obras podemos encontrar descripciones relativas a la práctica médica de la época e incluso de la medicina actual. Escribió entre 35 y 38 piezas teatrales, seis obras líricas y 154 sonetos (aproximadamente), en los que es posible encontrar poco más de 700 referencias a la medicina de su tiempo. Por tal motivo, diversos estudiosos han analizado los conceptos médicos en la literatura shakesperiana; algunos coinciden en que puede ser el resultado de su estudio, mientras que otros indican que Shakespeare repitió el conocimiento médico de algunos "hombres de saber" de su tiempo. Cabe mencionar que la sociedad londinense contemporánea al autor vivía en una ciudad sobrepoblada, nauseabunda, sexualmente promiscua y atestada de plagas y desechos, que continuamente era asediada por epidemias, cuyos tratamientos muchas veces ocasionaban un mal mayor a su beneficio (por ejemplo, el maquillaje de plomo). Algunos biógrafos dan crédito a la sífilis, el alcohol y la depresión como origen de la producción creativa de Shakespeare. Aun cuando un sinfín de autores se han dado a la tarea de examinar las alusiones sobre medicina en los relatos dramáticos de Shakespeare, Sigmund Freud (1856-1939) fue quizás el más importante; a lo largo de su carrera elaboró un análisis sustancial de las obras del dramaturgo inglés debido a su pasión por la literatura y hacia este artista.

PALABRAS CLAVE: Shakespeare. Neurología. Cognición. Psiquiatría.

\begin{abstract}
William Shakespeare (1564-1616) is with no doubt one of the most prolific literary artists of all times; in addition to being an eminent playwright, his works reflect arguments that express medical knowledge, allude to concise descriptions that are relevant to medical practice of that time, and even to current medicine. He wrote between 35 and 38 plays, six lyric works and 154 sonnets (approximately) where finding little more than 700 references to medicine of those days is possible. For this reason, different scholars have analyzed the medical concepts within the Shakespearean literature; some agree that these may be the result of studies of his own, while others suggest that Shakespeare repeated the medical knowledge of some "men of wisdom" of his time. It should be mentioned that the author's contemporary London society lived in an overpopulated, nauseating, sexually promiscuous city that was plagued by pests and waste and continually besieged by epidemics whose treatments many times caused greater evil than benefit (for example, lead-based make-up). Some biographers credit syphilis, alcohol,
\end{abstract}

Fecha de recepción: 26-02-2017

Fecha de aceptación: 24-09-2017

DOI: 10.24875/GMM.17003332
Gac Med Mex. 2018;154:613-616

Disponible en PubMed

www.gacetamedicademexico.com 
and depression as the source of Shakespeare's creative production. Despite the myriad of authors who have analyzed the insinuations about medicine in Shakespeare's dramatic accounts, Sigmund Freud (1856-1939) is perhaps the most important; throughout his career he carried out a substantial analysis of the English playwright's works owing to his passion for literature and for this author.

KEY WORDS: Shakespeare. Neurology. Cognition. Psychiatry.

William Shakespeare (1564-1616) vivió durante la edad de oro del drama inglés (fin del reinado de Elizabeth I e inicio de James I) y es sin duda uno de los artistas literarios más prolíficos de todos los tiempos., ${ }^{1,2}$ Además de su calidad literaria, sus obras expresan conocimiento médico: en ellas se encuentran descripciones relevantes de la práctica médica de la época e incluso de la medicina actual. ${ }^{1-4}$ El análisis descriptivo de algunos personajes permite distinguir en ellos trastornos de índole neurológica o psiquiátrica; muchos de los héroes y antihéroes de Shakespeare son ejemplos de demencia, parasomnia, parkinsonismo, epilepsia, migraña, parálisis e, incluso, enfermedades infecciosas. $^{5-9}$ A 400 años del deceso del Bardo de Avon, sus obras continúan siendo fuente de inspiración.

Es sorprendente la cantidad de datos clínicos en los trabajos de Shakespeare; escribió entre 35 y 38 piezas teatrales, seis obras líricas y 154 sonetos (aproximadamente), en los que es posible encontrar poco más de 700 referencias a la medicina de la época.,10-12 Por tal motivo, diversos investigadores han analizado los conceptos médicos en la literatura shakesperiana; algunos coinciden en que puede ser el resultado de su estudio, mientras que otros indican que Shakespeare repitió el conocimiento médico de algunos "hombres de saber" de su tiempo.9,13 Por ejemplo, según Gómes, ${ }^{1}$ la escena donde Hamlet sostiene el cráneo de Yorick, el bufón de la corte, recuerda un grabado de Andrés Vesalio (1514-1564); en la obra, la imagen es usada para meditar sobre el vínculo entre vida y muerte, así como el reconocimiento de la sabiduría adquirida a través de los antepasados.

Cabe mencionar que la sociedad londinense contemporánea al autor vivía en una ciudad sobrepoblada, nauseabunda, sexualmente promiscua y atestada de plagas y desechos, que continuamente se veía asediada por epidemias cuyos tratamientos, muchas veces ocasionaban un mal mayor a su beneficio (por ejemplo, el maquillaje de plomo)..$^{9,13-16}$ Dicho esto, algunos biógrafos dan crédito a la sífilis, al alcohol y a la depresión como origen de la creatividad de Shakespeare. ${ }^{17,18}$ Ross, ${ }^{17}$ a partir de la escritura y firma del Bardo y de las innumerables referencias a enfermedades de trasmisión sexual en sus textos, reforzó esta hipótesis:
Un diagnóstico unificador para el temblor de Shakespeare, la agitación y el aislamiento social es el envenenamiento por vapor de mercurio.

Es posible que el autor de Macbeth hubiese adquirido estos conocimientos a través de la vida diaria y la lectura, además de plasmar ciertos aspectos intrínsecos a la sociedad de la época.

Stompe et al. ${ }^{14}$ atribuyen la presencia de características clínicas concordantes con locura en los personajes ficticios de Otelo, Hamlet, el rey Lear y Macbeth, al entorno sociocultural de la época isabelina. Igualmente sugiere que, tras leer la obra es posible distinguir "melancolía" (término isabelino para la depresión), delirios y alucinaciones, así como esquizofrenia, que aún no había sido descrita como una enfermedad.

De igual manera, Montalt, ${ }^{4}$ en su serie de escritos, repasa los acontecimientos más simbólicos para la medicina que son mencionados en diferentes obras de Shakespeare. A la par, define los padecimientos de algunos personajes: depresión (Hamlet), epilepsia (Julio César), celotipia (Otelo), demencia (Lear), adicciones (Falstaff), escoliosis y psicopatía (Ricardo III).

Por otra parte, similar a lo que le sucede en Hamlet, el insomnio, el sonambulismo y las pesadillas se hacen evidentes en Macbeth durante el transcurso del quinto acto, incluso se recurre a la voz del médico como un recurso para la alusión directa:

-Una gran alteración de la naturaleza, recibir a la vez el beneficio del sueño y hacer las cosas de cuando se está despierto (Macbeth, acto V, escena 1). ${ }^{19}$

Høyersten ${ }^{20}$ centra su atención en las reacciones psicóticas que lady Macbeth sufre dormida tras matar a la vieja usurera y su hermana con un hacha:

-El barón Fife tenía una esposa: ¿y dónde está ahora? Qué, ¿no van a quedar nunca limpias estas manos?" (Macbeth, acto $\mathrm{V}$, escena 1). ${ }^{19}$

Sin embargo, es incuestionable que en la literatura shakesperiana Otelo es una de las mejores descripciones de los trastornos del sueño, ya que contiene una gran cantidad de alusiones a la noche, el sueño y las parasomnias:

...Las bendiciones de la noche os acompañen, amigos..." (Otelo, acto I, escena 2) $)^{19}$ 
De acuerdo con Dimsdale, ${ }^{21}$ Shakespeare era obviamente consciente de las parasomnias sexuales para haberlas descrito detalladamente en su tragedia. Paralelamente afirma que Otelo ofrece una imagen vívida de la privación del sueño como una herramienta para la persuasión.

Por su parte, Chiu ${ }^{9}$ subraya la celotipia que Otelo demuestra en gran parte de la obra; hace referencia al "síndrome de Otelo", denominación impuesta por el médico inglés John Todd a la condición psiquiátrica de los celos sexuales en un artículo en coautoría con K. Dewhurst titulado The Othello syndrome: a study in the psychopathology of sexual jealousy (1955).

A pesar del sinfín de autores que han analizado las insinuaciones sobre la medicina en los relatos dramáticos de Shakespeare, el alemán Sigmund Freud (1856-1939) es quizás el más importante, ya que debido a su pasión por la literatura y hacia el dramaturgo inglés, a lo largo de su carrera llevó a cabo análisis sustanciales de sus obras.

En sus escritos The interpretation of dreams (1900) y Psychopathetic characters on the stage (1905), Freud ${ }^{21}$ señaló que la literatura shakesperiana es un reflejo del "terror y la piedad" autocomplacientes para purgar las emociones y deseos más profundos. Freud criticó juiciosamente, mediante el psicoanálisis, lo expresado por Shakespeare en muchas de sus obras; puntualizó características importantes referentes a la psiquiatría y distinguió a Hamlet como un modelo clásico de una figura psicopática, quien sufría deseos reprimidos para desplazar a su padre (Oedipus Rex). 9,15,22

Acerca del resentimiento social que Ricardo III expresa abiertamente y quien se hace acreedor así mismo de una "excepción" que le permite reclamar privilegios sobre los demás debido a su aspecto disforme, Freud lo señala como una condición inherente al ser humano, que siente la necesidad de una excusa para reclamar la misma exención de algunas normas sociales. ${ }^{9}$

Del mismo modo, Freud elogió la representación que Ofelia ofrece para la "melancolía" en Hamlet. ${ }^{9} \mathrm{Sin}$ embargo, no fue el primero en puntualizar esta similitud. Fue el psiquíatra inglés Hugh Welch Diamond (1809-1886) quien introdujo la fotografía para el estudio del tratamiento de la enfermedad mental; su trabajo On the application of photography to the physiognomic and mental phenomena of insanity (1856) contiene una serie de calotipos de pacientes del Lunatic Asylum del condado de Surrey, a manera de representación de sus enfermedades. En su mayoría se trata de retratos de mujeres; al observarlas es posible encontrar a una de estas mujeres caracterizada como Ofelia, figura que sería adoptada como imagen de la locura femenina juvenil, la histeria y el colapso mental. ${ }^{23,24}$

A 400 años del deceso de Shakespeare, su dramaturgia y poesía son una continua fuente de inspiración no solo en el campo de la psicología y psiquiatría. El neurólogo francés Guillaume Benjamin-Amand-Duchenne (1806-1875) utilizó el realismo de las emociones percibidas en Macbeth para comprobar sus estudios, como lo plasmó en su escrito Mécanisme de la physionomie humaine ou analyse électro-physiologique des passions aplicable à la pratique des arts plastiques (1862), en el que a modo de atlas expuso una serie de ilustraciones acerca de las expresiones faciales. ${ }^{23,25-27}$ Las imágenes de Duchenne combinan arte, literatura y ciencia; empleó la fotografía, por su superioridad tecnológica, para plasmar los rostros y en algunas láminas (81, 82 y 83) se concentró en la recreación de pasajes de Macbeth. ${ }^{27}$ La intención de Duchenne fue reproducir los signos característicos de las efusiones violentas sobre el rostro de una joven actriz, quien caracterizaba a lady Macbeth, mediante estimulación eléctrica. ${ }^{23}$

Para Jean-Martin Charcot (1825-1893), la obra de Shakespeare representó una herramienta educativa para la enseñanza. Recurrentemente utilizó referencias shakesperianas en sus textos y sesiones de neurología; subrayó enfáticamente cómo la observación objetiva y la atención al detalle eran esenciales para el diagnóstico clínico experto. ${ }^{28,29}$ Comúnmente empleaba como ejemplo un personaje de Enrique VI, Say, quien a lo largo de la obra sufría temblores, lo que es evidenciado por Dick, el carnicero, quien le pregunta: “¿Por qué te estremeces?”, a lo que Say responde: “La parálisis, no el miedo me lo provoca". Charcot, a diferencia de sus contemporáneos, apuntaba el origen del temblor como no propio del envejecimiento..$^{30,31}$

Al explorar a fondo las referencias a enfermedades neurológicas es posible encontrar alusiones al parkinsonismo a través de la descripción de Shylock, un despiadado usurero en El mercader de Venecia. Al mismo tiempo, los grabados contemporáneos relacionados con el personaje expresan características clínicas concordantes con esta afección (hipomimia, camptocormia, pocket sign, entre otros).

Por su parte, la epilepsia (falling sickness) se menciona tres veces en forma directa e indirecta en las obras de Shakespeare: en Julio César (acto I, escena 2), Otelo (acto IV, escena 1) y, figurativamente, en El rey Lear (acto II, escena 2). ${ }^{32-35}$

- Se cayó en el mercado, y tenía espuma en la boca, y se quedó sin habla. (Julio César, Casca, acto I, escena 2). ${ }^{19}$ 
Aunque en Otelo se menciona de forma franca la enfermedad (epilepsy), para muchos autores, la situación circundante que se narra representa un episodio de síncope vasovagal. ${ }^{34}$ De igual forma, según Collado Vázquez, ${ }^{35}$ Bergfeldt registró en la literatura shakesperiana 18 desmayos que a veces se acompañan de sacudidas; sin embargo, él mismo los califica como síncopes vasovagales.

También es notable que Shakespeare tuviese conocimientos de anatomía avanzada, ya que en tres ocasiones se refiere a la piamadre:

-...estos se engendraron en el ventrículo de la memoria, alimentada en el seno de la piamadre. (Love's Labour's Lost, Holofornes, acto IV, escena 2). ${ }^{13}$

En años recientes, tras analizar profundamente los diálogos, Matthews ${ }^{5}$ propuso una nueva hipótesis: que el rey Lear sufría de demencia por cuerpos de Lewy; no obstante, existen pocas evidencias de la misma.

Finalmente, pocas obras reflejan a profundidad la extensión del conocimiento médico por parte Shakespeare. Para ahondar en este tema se recomienda The medical knowledge of Shakespeare (Bucknill, 1860), Shakespeare and medicine (Simpson, 1959) y The medical mind of Shakespeare (Kali, 1986).

Como comentario final, la imaginación a través de la medicina es un componente importante del mundo de Shakespeare y su tipo de escritura; es ampliamente aceptado que el Bardo de Avon era único en el conocimiento de la mente humana, a tal punto que tanto Freud como Mahon ${ }^{36,37}$ señalan la existencia de parapraxis en los diálogos, como un evento cuidadosamente premeditado que deja relucir un doble contexto y cuya finalidad es la exaltación del espectador.

Según Gómes, ${ }^{1}$ Moore encontró la creación de palabras a partir de la combinación de otras y de la sintaxis, lo que atribuyó a una "neurociencia". Recientemente, Keidel et al. ${ }^{38}$ intentaron explicar este hecho mediante estudios de neuroimagen; concluyeron que los cambios funcionales en la retórica utilizada por Shakespeare provocan un efecto de sorpresa que desencadena una reevaluación mental del proceso semántico y, a su vez, una activación más allá de las regiones clásicamente funcionales para las tareas típicas de lenguaje.

Por todo esto y más, es posible hablar de Shakespeare como un "neurólogo renacentista". Con su comprensión de la personalidad, el comportamiento y el subconsciente, ha dado forma a nuestra penetración de la mente humana a través de las edades. ${ }^{1}$

\section{Bibliografía}

1. Gómes-Da Mota M. Shakespeare's: his $450^{\text {th }}$ birth anniversary and his insights into neurology and cognition. Arq Neuro-Psiquiatr. 2015;73:359-361.

2. Fogan L. The neurology in Shakespeare. Arch Neurol. 1989;46:922-924.

3. Kail AC. The doctors in Shakespeare's plays. Part One. Aust Fam Physician. 1990;19:211-214.

4. Montalt V. Shakespeare y la medicina: dramaturgias del cuerpo y la mente. Panace@. 2015;16:73-77

5. Matthews BR. Portrayal of neurological illness and physicians in the works of Shakespeare. Front Neurol Neurosci. 2010;27:216-226.

6. Paciaroni M, Bogousslavsky J. William Shakespeare's neurology. Prog Brain Res. 2013;206:3-18.

7. Bogousslavsky J, Paciaroni M. Parkinsonism in poets and writers. Prog Brain Res. 2013;206:59-71.

8. Umanath S, Sarezky D, Finger S. Sleepwalking through history: medicine, arts, and courts of law. J Hist Neurosci. 2011;20:253-276.

9. Chiu C. Freud on Shakespeare: an approach to psychopathetic characters. Chang Gung Journal of Humanities and Social Sciences. 2012;5:33-56.

10. Payá E. Las enfermedades infecciosas en la obra de William Shakespeare. Rev Chil Infectol. 2013;30:660.

11. The Shakespeare Globe Trust. William Shakespeare. Londres: 2013.

12. Shakespeare: The Bard at the bedside. Lancet. 2016;387:1693.

13. Davis FM. Shakespeare's medical knowledge: how did he acquire it? The Oxfordian. 2000;3:45-58

14. Stompe T, Ritter K, Friedmann A. Die Gestaltungen des Wahnsinns bei William Shakespeare. Wien Klin Wochenschr. 2006:118:488.

15. Strachey J, Freud A, Strachey A, Tyson A. The standard edition of the complete psychological works of Sigmund Freud. Londres: Hogarth; 1953-1974.

16. Guijarro-Castro C, Estallo-Guijarro L. Cervantes y Shakespeare, dos neurólogos renacentistas. Rev Neurol. 2017;65:96.

17. Ross JJ. Shakespeare's chancre: did The Bard have syphilis? Clin Infect Dis. 2005; 40:399-404.

18. Hanlon V. Shakespeare's tremor and Orwell's cough: the medical lives of famous writers. CMAJ. 2014;186:E67.

19. Shakespeare W. Tragedias. España: Planeta; 1994

20. Høyersten JG. Legen, skjønnlitteraturen og den psykiatriske pasienten. Tidsskr Nor Laegeforen. 2005;125:3460-3463.

21. Dimsdale JE. Sleep in Othello. J Clin Sleep Med. 2009;5:280-281.

22. Freud S, Bunker HA. Psychopathic characters on the stage. The Tulane Drama Review. 1960;4:144-148.

23. Montilla J. llustraciones médicas de la locura femenina en el siglo XIX. En: Encuentro: artes por la integración. España: Diputación da Coruña; 2012.

24. Pérez, J. Women of the Surrey County Lunatic Asylum: first photographic portraits of mental illness (1852). Arch Womens Ment Health. 2016;19:201-202.

25. Heaton KW. Body-conscious Shakespeare: sensory disturbances in troubled characters. Med Humanit. 2011;37:97-102.

26. Fernández-Vázquez JM, Aziz-Jacobo J, Camacho-Galindo J. Guillaume Benjamín Amand Duchenne (1806-1871). El padre de la electrofisiología. Acta Ortop Mex. 2006;20:294-296.

27. Duchenne-De Boulogne GBA. Mécanisme de la physionomie humaine ou Analyse électro-physiologique de l'expression des passions applicable à la pratique des arts plastique. Francia: JB Ballière; 1876.

28. Gomes MM, Engelhardt E. Jean-Martin Charcot, father of modern neurology: an homage 120 years after his death. Arq Neuropsiquiatr. 2013;71:815-817.

29. Goetz CG. Shakespeare in Charcot's neurologic teaching. Arch Neurol. 1988;45:920-921.

30. Pahwa R, aLyons KE. Handbook of Parkinson's Disease. EE. UU.: CRC Press; 2013.

31. Finger S. Origins of neuroscience: a history of explorations into brain function. EE. UU.: MIT Press; 1994.

32. Breuer H. Images of epilepsy in Shakespeare. Medizinhist J. 2002;37:5-19.

33. Larner AJ. Neurological literature: Epilepsy. ACNR. 2007;7:16.

34. Bruschi F. Was Julius Caesar's epilepsy due to neurocysticercosis? Trends Parasitol. 2011;27:373-374.

35. Collado-Vázquez S, Carrillo JM. La epilepsia en la literatura, el cine y la televisión. Rev Neurol. 2012;55:431-442.

36. Mahon E. A parapraxis in Hamlet. A note on the aesthetic genius of William Shakespeare. Psychoanal Study Child. 1998;53:276-281.

37. Mahon E. Parapraxes in the plays of William Shakespeare. Psychoanal Study Child. 2000:55:335-370.

38. Keidel JL, Davis PM, González-Díaz V, Martín CD, Thierry G. How Shakespeare tempests the brain: neuroimaging insights. Cortex. 2013;49:913-919. 